ISSN: $2637-6628$

DOI: $10.32474 / 0 J N B D .2018 .01 .000107$

\title{
IgG4-Related Disease Misdiagnosed as Cholangiocarcinoma
}

\author{
El Hadary $\mathrm{HF}^{1 *}$, Dadour $\mathrm{NM}^{2}$, Ahmed $\mathrm{H}^{3}$ and Mo'nes $\mathrm{D}^{\mathbf{3}}$ \\ ${ }^{1}$ Consultant of Rheumatology \& Immunology, Cairo University, Egypt \\ ${ }^{2}$ Specialist of Rheumatology \& Rehabilitation, Egyptian Fellowship, Egypt \\ ${ }^{3}$ Resident of Rheumatology \& Rehabilitation, El Katib Hospital, Egypt
}

Received: 跙April 16, 2018; Published: 眥 April 24, 2018

*Corresponding author: Hala El Hadary, Consultant of Rheumatology \& Immunology, Kasr El-Ainy, School of Medicine, Cairo University, Egypt

\section{Abstract}

Immunoglobulin G4-related disease (IgG4-RD) is an increasingly recognized immune-mediated condition comprised of a collection of disorders that share particular pathologic, serologic, and clinical features [1,2]. These disorders were previously thought to be unrelated [3-5]. The commonly shared features include tumor-like swelling of involved organs, a lymphoplasmacytic infiltrate enriched in IgG4-positive plasma cells, and a variable degree of fibrosis that has a characteristic "storiform" pattern. In addition, elevated serum concentrations of IgG4 are found in 60 to 70 percent of patients with IgG4-RD.

IgG4-related sclerosing cholangitis (IgG4-SC) is a characteristic type of sclerosing cholangitis, with an unknown pathogenic mechanism. Patients with IgG4-SC display increased serum IgG4 levels [6] and dense infiltration of IgG4-positive plasma cells with extensive fibrosis in the bile duct wall [7]. Circular and symmetrical thickening of the bile duct wall is observed in the areas without stenosis that appear normal on cholangiography, as well as in the stenotic areas [8]. IgG4-SC has been recently recognized as an IgG4related disease. IgG4-SC is frequently associated with autoimmune pancreatitis (AIP). IgG4-related dacryoadenitis/sialadenitis and IgG4-related retroperitoneal fibrosis are also occasionally observed in IgG4-SC [9-12]. However, some IgG4-SC cases do not involve other organs. IgG4-SC is most common in elderly men. Obstructive jaundice is frequently observed in IgG4-SC.

A number of diseases, such as, Cystic fibrosis, Chronic obstructive Choledocholithiasis, Biliary strictures (secondary to surgical trauma, chronic pancreatitis), Anastomotic strictures in liver graft, Neoplasms (benign, malignant, metastatic), Infections, hypertonic saline instillation in the bile ducts, Post-traumatic sclerosing cholangitis, Systemic vasculitis, Amyloidosis, Radiation injury, Sarcoidosis, Systemic mastocytosis, Hypereosinophilic syndrome, Hodgkin's disease, may easily be confused with IgG4related sclerosing cholangitis, or coexist in a patient [13]. In this case, report 57 years male patient presented with jaundice, fatigue, weight loss, oral moniliasis and right sided neck swelling. He was misdiagnosed as Cholangiocarcinoma.

Keywords: IgG4-Related disease; Cholangiocarcinoma; Neck swelling; Jaundice

Abbreviations: IgG4-RD: Immunoglobulin G4-Related Disease; AIP: Auto Immune Pancreatitis; MRCP: Magnetic Resonance Cholangio Pancreatography; CT: Computed Tomography; ERCP: Endoscopic Retrograde Cholangio Pancreatography; PSC: Primary Sclerosing Cholangitis

\section{Introduction}

IgG4-related disease is a newly recognized fibro inflammatory disorder. Tumefactive lesions, storiform fibrosis, IgG4-positive plasma cells infiltration and frequent but not always elevated serum IgG4 level characterize it [14]. IgG4-related sclerosing cholangitis

(IgG4-SC) is the most common extra pancreatic manifestation of IgG4-related disease, and it has become the third distinct disease entity of sclerosing cholangitis [15]. The clinical and radiological abnormalities seen in IgG4-SC may resemble those seen in cholangiocarcinoma. IgG4-SC frequently keeps accompany with 
concurrent autoimmune pancreatitis (AIP). Only few cases were reported to be diagnosed with IgG4-SC in the absence of AIP, with a male preponderance [16].

\section{Case Report}

This is a 57 years old male patient, diabetic but not hypertensive, Presented with right submandibular swelling, epigastric fullness anorexia, jaundice, itching, fatigue, weight loss, oral moniliasis and Tea colored urine for 10 days, Laboratory tests showed normal white blood cell count, 5930/Ul, elevated serum bilirubin, total $6.3 \mathrm{mg} / \mathrm{dl}$ (0.4 - 1.4), direct $4.1 \mathrm{mg} / \mathrm{dl}(<0.4)$, high alanine aminotransferase (ALT) $222 \mathrm{U} / \mathrm{L}$ (3-30) and high aspartate amino transferase (AST) $114 \mathrm{U} / \mathrm{L}$ (10-35). Under the tentative diagnosis of obstructive jaundice. He underwent abdominal ultrasonography and dilatation of bilateral intrahepatic bile ducts was noted. Magnetic resonance cholangiopancreatography (MRCP) was performed. A short segment of stenosis in both hepatic ducts with marked poststenotic dilatation was revealed. Then, he underwent triphasic abdominal computed tomography (CT), which showed small hilar hepatic mass with dilated intra hepatic biliary radicals.

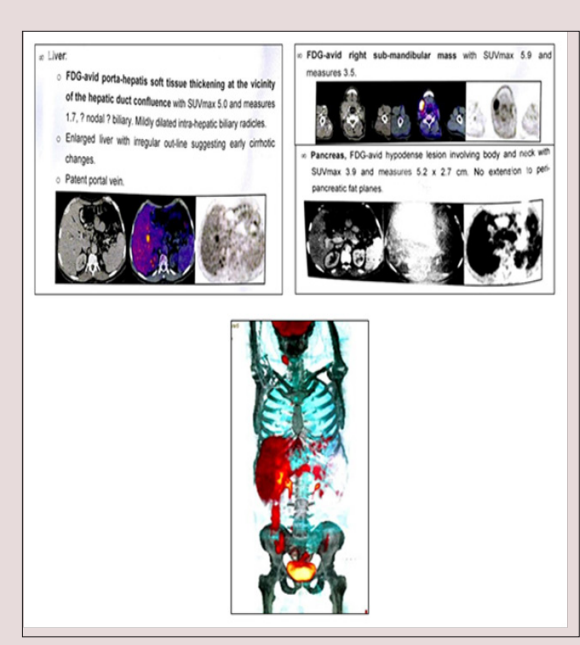

Figure 1: PET/CT Study.

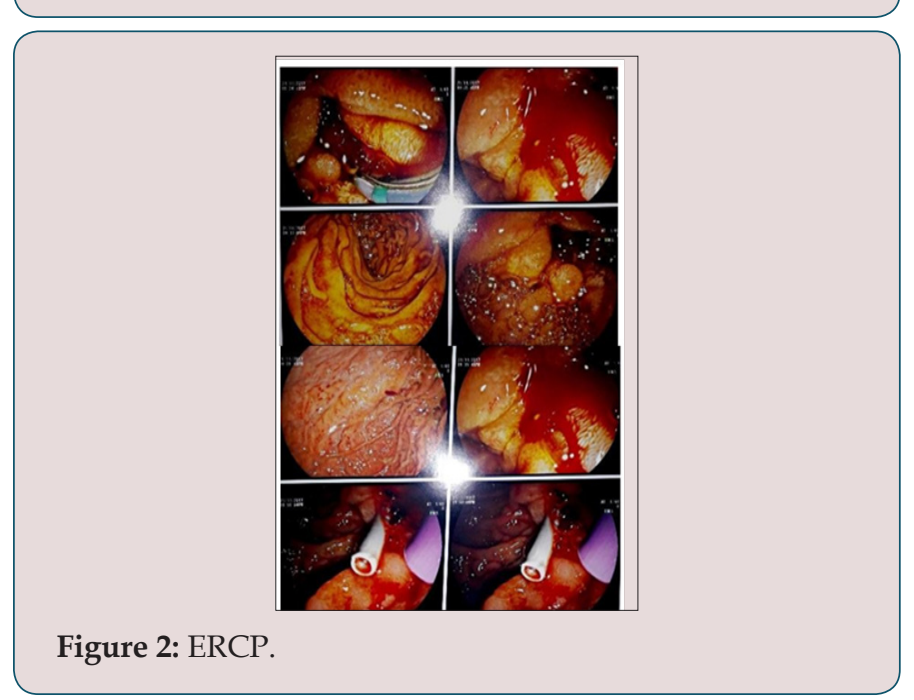

PET/CT study showed hypermetabolic pancreatic body lesion, hypermetabolic porta-hepatic soft tissue thickening and metabolically active right sub-mandibular mass (Figure 1). Cholangiocarcinoma was highly suspected and internal drainage was done with two stents by Endoscopic retrograde cholangiopancreatography (ERCP) (Figure 2). Pancreatic body mass biopsy showed chronic pancreatitis and inflammatory pseudo tumor. The tumor markers were checked and showed CEA $2.75 \mathrm{mg} /$ $\mathrm{ml}(<3.4)$ and AFP $6.2 \mathrm{mg} / \mathrm{ml}(<8)$. The serum total bilirubin was declined to $1.86 \mathrm{mg} / \mathrm{dl}$ after ERCP. Salivary gland excision biopsy was done which showed extensive fibrosis, macrophages and dens plasma cell infiltration, multinucleated giant cells of foreign body type and the plasma cell were positive for IgG4 monoclonal antibody with no evidence of malignancy. The IgG4-positive plasma cells account for 80 - 100 per high-power field despite normal total IgG and IgG4 serum level. The patient diagnosed as IgG4 related disease and received rituximab $1 \mathrm{~g}$ twice with 2 weeks interval in addition to oral prednisolone $40 \mathrm{mg} / \mathrm{d}$ which was tapered gradually with marked improvement clinically and laboratory.

\section{Discussion}

The IgG4-associated cholangitis (IAC) is one of the IgG4 associated sclerosing disease. In fact, the IgG4 associated sclerosing disease had been reported to involve many organs, causing IgG4 associated sclerosing pancreatitis, cholangitis, retroperitoneal fibrosis, sialadenitis, lymphadenopathy, thyroiditis, nephritis, pneumonia, prostatitis, and some inflammatory pseudotumors [17]. Overlapping of these IgG4 associated sclerosing diseases is common. They are characterized by an elevated serum IgG4, extensive IgG4-positive plasma cells and T-lymphocyte infiltration in the involved organs and well responded to steroid therapy. The pathogenesis of IgG4-associated sclerosing disease remains undetermined [18].

Diagnosis of IAC requires a high index of suspicion. The differential diagnoses include primary sclerosing cholangitis (PSC), cholangiocarcinoma, pancreatic cancer and benign traumatic biliary stricture. The cholangiographic appearance of IAC is not specific. The stricture of bile duct in IAC might be in lower end of common bile duct when combined with autoimmune pancreatitis (AIP). Some were multiple and may be in the intrahepatic or the hilar hepatic bile duct and very similar to that of Primary sclerosing cholangitis (PSC) [19-21]. When the stricture is solitary and had no other combined pancreatic disease, it will be difficult to differentiate from carcinoma. Our case had a stricture in hepatic duct and normal pancreas, which led to misdiagnosis of cholangiocarcinoma preoperatively.

The elevated serum IgG4 is a hallmark of IAC, but it is not diagnostic for the disease. Not all IAC cases have high serum IgG4 [22]. On the contrary, some cases of PSC and other diseases might have high serum IgG4. It is difficult to differentiate 
cholangiocarcinoma from IAC by present imaging studies [23]. Use of IgG4 immunostaining on cytology specimens is not recommended because the density of IgG4- positive cells in the tissue cannot be determined from these specimens. Mild tissue IgG4 immunostaining can occur in other diseases [24]. Therefore, endoscopic brush cytology could not help to make a diagnosis of IAC, but a malignant result of cytology could exclude IAC. Preoperative diagnosis is sometimes difficult, especially when serum IgG4 is not high. Histological examination of the surgical specimen is needed to make a final diagnosis in some rare cases. The optimal steroid treatment regimen of IAC is not defined. Most patients respond initially to steroids but relapse is not uncommon 17. In patients with IAC, careful observation for relapse of cholangitis or other possible IgG4 associated sclerosing diseases is mandatory both during and after withdrawal of the steroid therapy. Though surgery is not indicated in patients with IAC, surgery had been performed in a great proportion of patients for the difficulty in making a precise diagnosis preoperatively before.

\section{References}

1. Stone JH, Zen Y, Deshpande V (2012) IgG4-related disease. N Engl J Med 366(6): 539-551.

2. Kamisawa T, Zen Y, Pillai S, Stone JH (2015) IgG4-related disease. Lancet 385(9976): 1460-1471.

3. Kamisawa T, Funata N, Hayashi Y (2003) A new clinicopathological entity of IgG4-related autoimmune disease. J Gastroenterol 38(10): 982.

4. Khosroshahi A, Stone JH (2011) A clinical overview of IgG4-related systemic disease. Curr Opin Rheumatol 23(1): 57.

5. Okazaki K, Uchida K, Koyabu M (2011) Recent advances in the concept and diagnosis of autoimmune pancreatitis and IgG4-related disease. J Gastroenterol 46(3): 277-288.

6. Hamano H, Kawa S, Horiuchi A, Unno H, Furuya N, et al. (2001) High serum IgG4 concentrations in patients with sclerosing pancreatitis. N Engl J Med 344(10): 732-738.

7. Zen Y, Harada K, Sasaki M, Sato Y, Tsuneyama K, et al. (2004) IgG4related sclerosing cholangitis with and without hepatic inflammatory pseudotumor, and sclerosing pancreatitis-associated sclerosing cholangitis: Do they belong to a spectrum of sclerosing pancreatitis? Am J Surg Pathol 28(9): 1193-1203.

8. Naitoh I, Nakazawa T, Ohara H, Ando T, Hayashi K, et al. (2009) Endoscopic transpapillary intraductal ultrasonography and biopsy in the diagnosis of IgG4-related sclerosing cholangitis. J Gastroenterol 44(11):1147-1155.
9. Kamisawa T, Funata N, Hayashi Y, Eishi Y, Koike M, et al. (2003) A new clinicopathological entity of IgG4-related autoimmune disease. J Gastroenterol 38(10): 982-984.

10. Ohara H, Nakazawa T, Sano H, Ando T, Okamoto T, et al. (2005) Systemic extrapancreatic lesions associated with autoimmune pancreatitis. Pancreas 31(3): 232-237.

11. Hamano H, Arakura N, Muraki T, Ozaki Y, Kiyosawa K, et al. (2006) Prevalence and distribution of extrapancreatic lesions complicating autoimmune pancreatitis. J Gastroenterol 41(12): 1197-1205.

12. Naitoh I, Nakazawa T, Ohara H, Ando T, Hayashi K, et al. (2010) Clinical significance of extrapancreatic lesions in autoimmune pancreatitis. Pancreas 39(1): e1-e5.

13. Takahiro Nakazawa, Itaru Naitoh, Kazuki Hayashi, Katsuyuki Miyabe, Shuya Simizu, et al. (2013) Diagnosis of IgG4-related sclerosing cholangitis. World J Gastroenterol 19(43): 7661-7670.

14. Stone J, Zen Y (2012) IgG4-related disease. N Engl J Med 366: 539-551.

15. Nakazawa T, Naitoh I, Hayashi K (2013) Diagnosis of IgG4-related sclerosing cholangitis. World J Gastroenterol 19(43): 7661-7670.

16. Lin J, Cummings OW, Greenson JK (2015) IgG4-related sclerosing cholangitis in the absence of autoimmune pancreatitis mimicking extrahepatic cholangiocarcinoma. Scand J Gastroenterol 50(4): 447-453.

17. Terumi Kamisawa, Atsutake Okamoto (2008) IgG4-related sclerosing disease. World J Gastroenterol 14: 3948-3955.

18. Hsien Ping Lin, Kwok Ting Lin, Wei Chi Ho, Chi Bing Chen, Chen Yun Kuo, et al. (2013) IgG4-associated Cholangitis Mimicking CholangiocarcinomaReport of A Case. J Intern Med Taiwan 24: 137-141.

19. Webster GJ, Pereira SP, Chapman RW (2009) Autoimmune pancreatitis/ IgG4- associated cholangitis and primary sclerosing cholangitis Overlapping or separate diseases? J Hepatol 51(2): 398-402.

20. Nakazawa T, Ohara H, Sano H (2005) Clinical differences between primary sclerosing cholangitis and sclerosing cholangitis with autoimmune pancreatitis. Pancreas 30(1): 20-25.

21. Kamisawa T, Egawa N, Tsuruta K (2005) Primary sclerosing cholangitis may be overestimated in Japan. J Gastroenterol 40(3): 318-319.

22. Hussain R, Poindexter RW, Ottesen EA (1992) Control of allergic reactivity in human filariasis. Predominant localization of blocking antibody to the IgG4 subclass. J Immunol 148(9): 2731-2737.

23. Daniel TM Chung, CN Tang, Eric CH Lai (2010) ImmunoglobulinG4Associated sclerosing cholangitis mimickingcholangiocarcinoma. Hong Kong Med J 16(2): 149-152.

24. Ghazale A, Chari ST, Zhang L (2008) ImmunoglobulinG4-Associated cholangitis: clinical profile and response to therapy. Gastroenterology 134: 706-715.

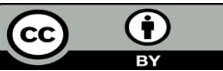

This work is licensed under Creative Commons Attribution 4.0 License

To Submit Your Article Click Here:

Submit Article

\section{OJNBD}

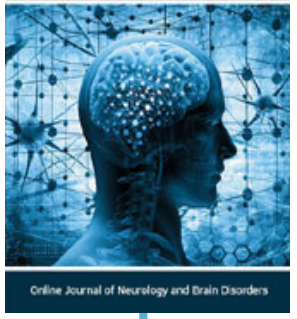

\section{Online Journal of Neurology} and Brain Disorders

\section{Assets of Publishing with us}

- Global archiving of articles

- Immediate, unrestricted online access

- Rigorous Peer Review Process

- Authors Retain Copyrights

- Unique DOI for all articles 